\title{
Dass eine Familie weiß, wo sie was an Hilfe kriegen kann, wenn sie Hilfe braucht, das finde ich wichtig - Sozialräumliche Vernetzung von Kitas in der Migrationsgesellschaft und die Rolle der Leitungen
}

\author{
Tanja Salem (D) - Drorit Lengyel
}

Online publiziert: 8. September 2020

(C) Der/die Autor(en) 2020

Zusammenfassung In vielen Regionen der Migrationsgesellschaft Deutschland ist Superdiversität Normalität für Kitas. Aufgabe von Kitas ist es, an den heterogenen Sozialisationserfahrungen und Bildungsvoraussetzungen von Kindern anzuknüpfen und ihre Arbeit sozialräumlich auszurichten, um Bildungsmöglichkeiten für Kinder (und Familien) zu eröffnen und zu erweitern. Im Anschluss an theoretische Konzepte zu Raum im Kontext von Bildung und die Rolle von Kita-Leitungen für die sozialräumliche Vernetzung in der Migrationsgesellschaft, geht die vorliegende empirische Studie den Zusammenhängen zwischen sozialstrukturellen und -kulturellen Bedingungen in und im Umfeld der Kita, der Vernetzung im Sozialraum sowie der Rolle der Kita-Leitungen hierfür nach.

Schlüsselwörter Kita · Sozialräumliche Vernetzung · Leitungen · Migrationsgesellschaft

Dr. T. Salem $(\bowtie) \cdot$ Prof. Dr. D. Lengyel

Fakultät für Erziehungswissenschaft, Allgemeine, Interkulturelle und International Vergleichende Erziehungswissenschaft sowie Pädagogische Psychologie, EW 1, Universität Hamburg,

Von-Melle-Park 8, 20146 Hamburg, Deutschland

E-Mail: tanja.salem@uni-hamburg.de

Prof. Dr. D. Lengyel

E-Mail: drorit.lengyel@uni-hamburg.de 


\title{
I think it is important for a family to know where they can get help when they need it-Social-spatial cooperation of ECEC institutions in the migration society and the role of leadership
}

\begin{abstract}
Social, ethnic and linguistic diversity is the norm in the migration society Germany, particularly in institutions of early childhood education and care. Childcare centres are legally required to take into account the social living environment of families, to base their educational offers on the diverse educational prerequisites and needs of children, and to cooperate with institutions in their neighbourhood. This study draws on theories on 'space and education' and leadership in early childhood education and care. It examines how leadership and cooperation relate to sociostructural and sociocultural conditions in childcare centres and the local area where they are based.
\end{abstract}

Keywords ECEC institutions · Cooperation · Neighbourhood · Leadership · Migration society

\section{Sozialräumliche Vernetzung in der Migrationsgesellschaft - ein Auftrag für alle Kitas}

Deutschland ist eine Migrationsgesellschaft. Superdiversität (Vertovec 2007), die sich auf die Diversifizierung von Diversität im Kontext von Migration bezieht, ist in vielen Regionen Deutschlands Normalität für Kitas. Sie betrifft Sozialisationserfahrungen und Bildungsvoraussetzungen von Kindern und drückt sich in sozialstrukturellen und sozialkulturellen Bedingungen aus, etwa in einer zwei- oder mehrsprachigen Sozialisation, Formen des familialen Zusammenlebens, Lebensstilen und (sozioökonomischen) Lebenslagen, Einbindungen in unterschiedliche Communities, weltanschaulichen Orientierungen, ethnischen Hintergründen und rechtlichen Status (Salem et al. 2020).

Nach dem Kinder- und Jugendhilfegesetz (KJHG/SGB VIII) ist in Kitas die ethnische Herkunft von Kindern neben sozialen und kulturellen Bedürfnissen sowie religiösen Orientierungen $(\S 9)$ bei der Förderung der geistigen, körperlichen, sozialen und emotionalen Entwicklung zu berücksichtigen ( $\$ 22$ [3]). Nach dem KJHG ist es außerdem Aufgabe von Kitas, mit anderen kinder- und familienbezogenen Institutionen und Initiativen im Gemeinwesen, insbesondere solchen der Familienbildung und -beratung, zusammenzuarbeiten ( $\$ 22 \mathrm{a}[2] 2$.) und das Angebot pädagogisch sowie organisatorisch an den Bedürfnissen der Kinder und ihrer Familien zu orientieren ( $\$ 22 \mathrm{a}$ [3]). Der Gesetzgeber sieht also vor, die pädagogische Arbeit den Sozialisationserfahrungen und Bildungsvoraussetzungen sowie Bedürfnissen von Kindern und Familien entsprechend zu gestalten und sie sozialräumlich auszurichten (Bundesministerium für Familie, Senioren, Frauen und Jugend 2020). Aktuell sollen Eltern-Kind-Zentren oder Familienzentren verstärkt dazu beitragen, diesen Auftrag umzusetzen; gleichwohl besteht er für alle Kitas (Jares 2019).

Es fällt in den Aufgabenbereich von Kita-Leitungen, Brücken zwischen der Kita und dem Sozialraum zu schlagen und Kooperationen mit Instanzen aufzubauen, die 
für die Erziehung, Bildung und Betreuung von Kindern sowie die Unterstützung von Familien relevant sind (Jung und Gels 2019; Strehmel und Ulber 2014). Ihnen kommt damit eine tragende Rolle dafür zu, Bildungsmöglichkeiten für Kinder und ihre Familien in der Migrationsgesellschaft zu eröffnen bzw. zu erweitern und damit zu gleichen Bildungschancen aller Kinder beizutragen.

Wie jedoch Kitas angesichts migrationsgesellschaftlicher Bedingungen sozialräumlich vernetzt sind, welche Bedeutung den sozialstrukturellen und -kulturellen Merkmalen der Kitas und der Sozialräume für die Vernetzung zukommt und wie Leitungen ihre Rolle für diese wahrnehmen, stellt eine Forschungslücke dar. Der vorliegende Beitrag greift dieses Desiderat auf.

Im nächsten Abschnitt werden Dimensionen pädagogischer Qualität im Kontext der Migrationsgesellschaft vorgestellt. Darauf folgt die Darstellung von Konzepten zu Raum und Bildung, woraufhin die Rolle von Kita-Leitungen für die sozialräumliche Vernetzung in der Migrationsgesellschaft behandelt wird. Hieran anschließend werden die Forschungsfragen präsentiert und das forschungsmethodische Vorgehen skizziert. Sodann werden die Ergebnisse der Studie vorgestellt. Der Beitrag schließt mit der Diskussion der Ergebnisse.

\section{Theoretische Bezüge und empirische Erkenntnisse}

\subsection{Pädagogische Qualität in der Migrationsgesellschaft: eine Annäherung}

Gängige Modelle pädagogischer Qualität stellen die Wechselbeziehungen der Dimensionen Struktur-, Orientierungs- und Prozessqualität, Organisations- und Managementqualität und Kontextqualität in den Mittelpunkt (z. B. Strehmel und Ulber 2014). Nach Stamm und Edelmann (2013) unterliegt ihnen ein ethnozentristisches Verständnis von Normalität, das der Diversität von Kindern nicht gerecht wird und damit Bildungsungleichheit reproduziert. Daher seien diese Modelle sowie ihre Umsetzung in der Migrationsgesellschaft kritisch zu prüfen. Zudem sind Machtverhältnisse bzw. -asymmetrien zwischen Individuen und auch unterschiedlichen (Status-)Gruppen und hegemoniale Diskurse zu berücksichtigen. Im Anschluss an diese Kritik und angesichts der superdiversen Migrationsgesellschaft werden für die vorliegende Studie die Dimensionen pädagogischer Qualität um einige migrationsspezifische Aspekte weiter ausdifferenziert (ausführlich siehe Salem et al. 2020):

Ziel frühkindlicher Bildung in der Migrationsgesellschaft ist es, Diversität von Kindern und Familien zu akzeptieren und sie - in dem Sinne, dass jedes Kind und jede Familie einzigartig ist - zum Ausgangspunkt des pädagogischen Handelns zu nehmen.

In der Prozessdimension ist sprachliche Bildung als wichtiges Handlungsfeld frühkindlicher Bildung in der Migrationsgesellschaft zu verorten. Die sprachlichkulturelle Diversität stellt dabei eine wesentliche Bedingung für sprachliche Bildungsprozesse dar, die es in sozialen Prozessen so zu berücksichtigen gilt, dass Kinder davon einen Nutzen für ihre sprachliche Entwicklung erfahren. In der Orientierungsdimension sind unter Berücksichtigung migrationsbedingter Diversität bei- 
spielsweise die Einstellungen gegenüber mehrsprachigem Aufwachsen anzusiedeln, aber auch Erziehungsvorstellungen der unterschiedlichen Akteure in Kitas.

Raumbezüge finden sich in der Strukturdimension und der Kontextdimension pädagogischer Qualität. Die Kontextdimension beinhaltet nach Strehmel und Ulber (2014) Merkmale des sozialen Umfeldes wie die sozioökonomische Situation der Familien und damit verbundene Ressourcen und Belastungen sowie das Vorhandensein bildungsrelevanter und familienunterstützender bzw. -ergänzender Institutionen im Stadtteil. Die Strukturdimension hingegen umfasst unter anderem die materielle (z.B. Spielmaterial, mehrsprachige Bücher) und die personelle Ausstattung (z.B. Anzahl und Qualifikation der Fachkräfte) in Kitas, die sozialstrukturellen und sozialkulturellen Bedingungen in der Kita (u. a. den Migrationshintergrund von Kindern, gesprochene Sprachen der Kinder und Fachkräfte) und den Erzieherinnen-KindSchlüssel. Zudem beinhaltet die Dimension Kooperation mit Instanzen außerhalb der Kita. Im Kontext der Migrationsgesellschaft ist die Vernetzung mit Instanzen wichtig, die sprachliche Bildungsprozesse unterstützen können. Darüber hinaus trägt die Zusammenarbeit mit migrationsspezifischen Einrichtungen wie Migrantenselbstorganisationen, Elterninitiativen und Dolmetscherdiensten dazu bei, die Teilhabe von Kindern und Eltern an Bildung zu ermöglichen und ihre Bildungsmöglichkeiten zu erweitern.

Basis für eine sozialräumliche Vernetzung ist, dass frühpädagogische Fachkräfte und Kita-Leitungen die Lebenslagen der Familien kennen und daran anknüpfend prüfen, welche sozialen Einrichtungen und Projekte im Sozialraum ansässig sind und inwieweit eine Zusammenarbeit mit ihnen sinnvoll und notwendig ist.

Die raumbezogenen Dimensionen pädagogischer Qualität fokussieren insbesondere materielle und sozialstrukturelle Bedingungen in einer Kita und in ihrem Umfeld. Raumtheoretische Konzepte erweitern diese Perspektive und berücksichtigen dabei gesellschaftliche Machtverhältnisse bzw. -asymmetrien und hegemoniale Diskurse.

\subsection{Das Konzept „Raum“ im Kontext von Bildung}

In den vergangenen Jahren hat eine raumtheoretische Perspektive in der Erziehungswissenschaft sowie der Pädagogik der frühen Kindheit (Jares 2019; Jung und Gels 2019) an Beachtung gewonnen, die sich auf einen relationalen Raumbegriff in Anlehnung an Martina Löw (2001) beruft.

Dirks und Kessl (2012) stellen im Anschluss an die Raumtheorie von Löw (2001) und raum(re)produktionstheoretische Ansätze räumliche Bedingungen im Umfeld von pädagogischen Institutionen, diese selbst und ihre Nutzerinnen und Nutzer sowie Fachkräfte (Akteure) als (Re)Produzentinnen und -produzenten von Räumen und die Praktiken letzterer in den Mittelpunkt ihrer Betrachtung. Drei Dimensionen sind in diesem Zusammenhang für die (Re)Produktion von Raum im Kontext von Bildung und Erziehung sowie sozialräumlicher Vernetzung relevant: Raum der Praktiken, Repräsentationen des Raumes und Raum der Repräsentation.

Unter Raum der Praktiken wird gefasst, wie das Materielle, physisch-sinnlich Fassbare und Wahrnehmbare (also etwa die Ausstattung der Kita mit [mehrsprachigen] Büchern und Material) das Handeln der Akteure beeinflusst und wie sie 
es (re)produzieren. Die Repräsentationen des Raumes meint den Diskurs über den Raum (sprachliche, grafische, symbolische Darstellungen und Markierungen), der (politische) Legitimität besitzt und sich z. B. niederschlägt in Sozial- und Bildungsatlanten, in denen sozialstrukturelle und -kulturelle Merkmale (wie der Anteil von Kindern mit einer anderen Erstsprache als Deutsch und der Sozialleistungsbezug der Bevölkerung) festgehalten sind. Der Raum der Repräsentation greift die Wahrnehmungen und das Erleben des Raumes auf, wodurch Raum (re)produziert wird.

Auch Stošić (2012), die sich ebenfalls auf Löw (2001) beruft, entwickelt ein bildungsbezogenes Raumkonzept, das das sog. Spacing sowie sog. Syntheseleistungen (s. unten) umfasst. Spacing ist ,,das Anordnen der sozialen Güter, Menschen (Lebewesen) an konkreten Orten [...], das Errichten, Bauen, Positionieren“ (Stošić 2012, S. 16). So werden etwa Einrichtungen der Familienberatung an einem Ort durch die zuständigen Behörden und/oder die Zivilgesellschaft platziert. Hierbei spielen das Verfügen über Ressourcen sowie gesellschaftliche Herrschafts- und Machtverhältnisse eine Rolle (siehe dazu auch Kessl und Reutlinger 2010). Die Bevölkerungszusammensetzung in Stadteilen weist durch eine raumtheoretische Brille auf die ,Platzierung von Menschen“ (Stošić 2012), etwa durch die Stadtplanung im Zuge der Arbeitsmigration in den 1960er/70er Jahren oder der verstärkten Fluchtmigration 2014/15, in traditionellen Arbeitervierteln bzw. städtischen Randbezirken. Aber auch die Selbstplatzierung vor dem Hintergrund transnationaler sozialer Netzwerke ist hier zu verorten, über die Menschen im Falle von Migration an Orte ziehen, in denen lebensrelevante soziale Bezüge und Communities bestehen. Diese Platzierung bildet sich ab in sozialstrukturellen und -kulturellen Merkmalen eines Ortes und spiegelt sich in der Regel, aber nicht zwangsläufig, auch in Bildungseinrichtungen.

Neben dem Spacing rücken in Stošićs Raumkonzept Syntheseleistungen in den Blick. Diese sind Verknüpfungen von sozialen Gütern, die von Menschen auf der Basis von Wahrnehmungs-, Erinnerungs- und Vorstellungsprozessen vorgenommen werden. Dabei sind die Syntheseleistungen gleichzeitig ,gesellschaftlich durch Raumvorstellungen, institutionalisierte Raumkonstruktionen und den klassen- und geschlechtsspezifischen Habitus vorstrukturiert" (Stošić 2012, S. 16). Durch Syntheseleistungen entsteht ein (Bildungs-)Raum. Hiermit geraten auch Relationen zwischen Institutionen in den Fokus, also Konkurrenz- und Kooperationsbeziehungen. Erst durch Syntheseleistungen wird also aus ,platzierten' sozialen Gütern und Menschen ein Bildungsraum konstituiert, der nach Stošić (2012, S. 20) ein „relevante[r] Faktor für Bildungsprozesse“ ist.

Kessl und Reutlinger (2010) folgend ist sozialräumlich orientierte Arbeit von Kitas damit nicht nur eine quartiers- oder stadtteilbezogene, sondern auch eine (sozial-)politische. Träger und politische Verantwortliche schaffen die Voraussetzungen für eine frühkindliche Bildung, die sich am Sozialraum orientiert. Gleichzeitig tragen Leitungs- und Fachkräfte die Verantwortung dafür, solch eine Arbeit zu realisieren. Hierfür bedarf es einer ,reflexiven räumlichen Haltung“ (ebd., S. 126) der Professionellen.

Zentral für die Konstituierung von Bildungsräumen und damit der sozialräumlichen Vernetzung ist die Wahrnehmung der Akteure in Kitas. Kita-Leitungen wird eine bedeutende Rolle für die Gestaltung frühkindlicher Bildung, was sozialräumliche Vernetzung umfasst, in Kitas zugeschrieben. Im nächsten Abschnitt gehen wir 
der Annahme nach, dass die Wahrnehmungen - und darauf basierendes Handeln von Leitungskräften von besonderer Bedeutung für die sozialräumliche Vernetzung sind.

\subsection{Zur Rolle der Leitungen}

Der Leitung von Kitas wie auch der Funktion(en) von Kita-Leitungen selbst wird in der deutschsprachigen Forschung in den vergangenen Jahren vermehrt Aufmerksamkeit geschenkt (Ballaschk und Anders 2015). Gleichwohl gibt es international eine längere Forschungstradition im Hinblick darauf, Leitung (leadership) und die Funktion(en) von Leitungskräften in den gesamtgesellschaftlichen Kontext zu stellen.

Das Konzept „contextually defined leadership“ von Hujala (2013) fasst, dass die Bedingungen und Interaktionen zwischen den verschiedenen Akteuren in Kitas wie Leitungen, Fachkräften und Familien, die unter je spezifischen Bedingungen leben, leadership beeinflussen. Aber auch die (lokale) Administration und Träger sowie gesellschaftliche Werte und Ideologien wirken hierauf. Nach Hujala (2013) ist leadership ein sozial konstruiertes, situatives und interpretatives Phänomen: „The situational system means that leadership is influenced by social situations and by the operational environment as well as by expectations and traditions of the society (ebd., S. 54)“. Ziele frühkindlicher Bildung stellen den Horizont dar, vor dem Leitungen handeln. Diese in die Praxis zu übersetzen, Strukturen und Prozesse (zwischen Akteuren in und mit dem Umfeld der Einrichtungen) entsprechend zu gestalten, ist eine Kernaufgabe der Leitungen.

Auch Hard et al. (2013) konstatieren, dass die Leitung von Kitas im Kontext gesellschaftlicher Bedingungen in einer globalisierten Welt zu sehen ist. Sie argumentieren, dass Kita-Leitungen eine zentrale Funktion zukommt, um bestehende gesellschaftliche Macht- und Ungleichheitsverhältnisse zu verändern.

Leitungen besitzen so ,a moral and social obligation to support equity for learners of various racial, gender, socio-economic and cultural backgrounds, sexual orientations and divers abilities“ (ebd., S. 328). Hard et al. (2013) sehen Leitungen als ,architects and builders“ (ebd., S. 328) einer neuen gesellschaftlichen Ordnung an, in der ,traditionell benachteiligte Personen“ die gleichen Bildungschancen haben wie die ,traditionell bevorteilten“ (ebd., S. 328). Ferner bezeichnen sie Leitungen als ,Brücken“ (ebd., S. 328) zwischen der Einrichtung und dem lokalen wie dem gesellschaftlichen Kontext in einer globalisierten Welt; sie müssen den lokalen Kontext und die Bedingungen darüber hinaus im Blick haben, um einen Beitrag zur Veränderung bestehender gesellschaftlicher (Ungleichheits-)Verhältnisse zu leisten.

Somit erscheinen Leitungen als wichtige Akteure dafür, dass in Kitas migrationsbedingte Diversität berücksichtigt und Bildungsziele, die sich an den Bedarfen und Bedürfnissen aller Kinder in der Migrationsgesellschaft orientieren, verfolgt und umgesetzt werden. Gleichzeitig fällt es maßgeblich den Kita-Leitungen zu, ,ihre ‘ Kita im Sozialraum zu verorten und Kooperationen mit Instanzen zu initiieren, die für die Erziehung und Bildung der Kinder und Unterstützung ihrer Familien relevant erscheinen. In einem größeren Zusammenhang betrachtet, können Leitungen zur 
Veränderung bestehender gesellschaftlicher Macht- und Ungleichheitsverhältnisse beitragen.

Theoretischen Konzepten zur Rolle der Kita-Leitung und zu Raum folgend, sind die Wahrnehmungen von Leitungen ihrer Einrichtung (z. B. strukturelle Bedingungen, Nutzerinnen und Nutzer) sowie des Sozialraums zentral für ihr Handeln in Bezug auf sozialräumliche Vernetzung. Allerdings sind empirische Studien zur Rolle von Kita-Leitungen für sozialräumliche Vernetzung rar. Im Hinblick auf die Kooperation zwischen Kitas und Grundschulen liegen Ergebnisse zur Rolle und Bedeutung der Leitungskräfte beider Institutionen aus Sicht von pädagogischen Fachkräften und Lehrkräften vor (z. B. Rathmer 2012). Die Studie von Salem (2018) zur Kooperation zwischen Kitas und Grundschulen im Kontext sprachlicher Bildung zeigte, dass Leitungen aus Sicht von Fach- und von Lehrkräften sowie von Leitungskräften selbst innerinstitutionell die Möglichkeiten für die institutionenübergreifende Kooperation schaffen. Die befragten Akteure weisen Leitungen eine zentrale Rolle hinsichtlich der Initiierung der Kooperation und der Übernahme von organisatorischen Aufgaben zu, die unter anderem das Zurverfügungstellen von Ressourcen (z. B. Zeit, Material) beinhalten. Zudem schaffen sie den inhaltlichen Rahmen für die Kooperation, indem sie innerinstitutionell Schwerpunkte in der Bildungsarbeit setzen, die Anknüpfungspunkte für die Kooperation darstellen. Die Wahrnehmungen der Leitungen spielen ebenfalls eine Rolle für die Kooperation: Eine geteilte Wahrnehmung von Handlungsbedarfen und geteilte Ziele sowie der persönliche Kontakt der Leitungskräfte begünstigen eine Institutionalisierung der Kooperation.

\section{Forschungsfragen}

Wie in den vorangegangenen Abschnitten dargestellt, wird Leitungen eine tragende Rolle im Hinblick auf die Gestaltung frühkindlicher Bildung - orientiert an den gesellschaftlichen Zielen frühkindlicher Bildung - in der Kita zugeschrieben (und bereits empirisch untermauert). Die Untersuchung der sozialräumlichen Vernetzung der Einrichtung mit ihrem Umfeld oder auch Sozialraum und die Rolle der Leitungen hierfür hingegen stellt ein Desiderat dar.

Dass die sozialstrukturelle und sozialkulturelle Zusammensetzung eines Stadtteils bzw. einer Kita (Repräsentationen des Raums nach Dirks und Kessl (2012) bzw. Spacing nach Stošić) Einfluss auf sozialräumliche Vernetzung besitzt, ist aus raumtheoretischer bzw. raum(re)produktionstheoretischer Perspektive nicht nur auf ,harte Fakten', die beispielsweise im Sozialatlas präsentiert werden, oder die vorhandenen Bildungsinstitutionen in einem Stadtteil zurückzuführen, sondern auch darauf, wie Akteure in Kitas diese Bedingungen wahrnehmen, wie sie auf dieser Basis handeln und was daraus für die Gestaltung von Bildungsprozessen folgt. $\mathrm{Ob}$ z. B. Einrichtungen der Familienberatung oder Migrantenselbstorganisationen einen Bestandteil des Bildungsraums einer Kita darstellen und ob Kooperationen eingegangen werden, hängt von den Syntheseleistungen (in Anlehnung an Stošić bzw. Raum der Repräsentation nach Dirks und Kessl 2012) etwa der Leitungskräfte ab, die sie auf Basis von Wahrnehmungs-, Erinnerungs- und Vorstellungsprozessen sowie gesellschaftlichen Vorstrukturierungen vollziehen. 
Vor diesem Hintergrund geht der Beitrag den Fragen nach (a) welche Bedeutung der sozialstrukturellen und sozialkulturellen Zusammensetzung des Stadtteils und der Kita für die sozialräumliche Vernetzung im migrationsgesellschaftlichen Kontext und (b) welche Rolle den Leitungskräften hierfür zukommt. Dabei stehen die Wahrnehmungen und subjektiven Sichtweisen von Leitungskräften im Mittelpunkt. Unter Bezugnahme auf die hier dargelegten theoretischen Vorstellungen und bisherigen empirischen Erkenntnisse, nehmen wir an, dass die beteiligten Kitas mit Partnern kooperieren, die im migrationsgesellschaftlichen Kontext relevant sind (z. B. hinsichtlich der sprachlichen Bildung), und dass die Leitungen eine aktive Rolle bei der Vernetzung übernehmen.

\section{Forschungsmethodisches Vorgehen}

Diese Forschungsfragen sind Teil eines interdisziplinären BMBF-Verbundprojekts (Laufzeit: 2018-2021). Das Projekt wird in sechs Hamburger Kitas unterschiedlicher Träger durchgeführt, in denen der Anteil von Kindern mit Migrationshintergrund bei ca. $40 \%$ und mehr liegt. Gesamtziel ist es, aus interkulturell-erziehungswissenschaftlicher und linguistischer Perspektive anhand von qualitativen Fallstudien das Zusammenspiel von sprachbezogener Interaktionsqualität und sprachbildungsrelevantem Wissen, Wahrnehmungs-, Deutungs- und Handlungsplanungskompetenz pädagogischer Fachkräfte (Fröhlich-Gildhoff et al. 2011) sowie einrichtungsinternen und -externen Bedingungen in der Migrationsgesellschaft zu rekonstruieren.

Materialgrundlage für diesen Beitrag stellen qualitative leitfadengestützte Interviews zu einrichtungsinternen und sozialräumlichen Bedingungen dar, die im Januar und Februar 2019 mit den Leitungskräften der sechs Kitas geführt wurden. In den Interviews wurde neben der Thematisierung des Aufgabenspektrums der Leitungen die sprachliche Bildung in der Kita beleuchtet, die Qualitätsentwicklung und -sicherung, die Wahrnehmung des Stadtteils und deren Bedeutung für die pädagogische Arbeit, die Vernetzung im Sozialraum (allgemein und auf sprachliche Bildung bezogen), die im Interview mithilfe einer Netzwerkkarte (Hollstein und Pfeffer 2010) erfasst wurde, sowie die Rolle der Leitung für die jeweiligen Bereiche. Die Interviews wurden digital aufgezeichnet und nach einfachen inhaltlich-semantischen Regeln transkribiert (Dresing und Pehl 2015). Zusätzlich wurden Daten zu Strukturmerkmalen (z. B. Anteil Kinder mit Migrationshintergrund, von Kindern und Fachkräften gesprochene Sprachen, Kooperationen) der jeweiligen Kita mit einem standardisierten Fragebogen erhoben, den die Leitungskräfte ausfüllten (Repräsentationen des Raums). Diese wurden deskriptiv ausgewertet.

Die Interviewdaten wurden mit MAXQDA mittels der qualitativen inhaltlichstrukturierenden Inhaltsanalyse (Kuckartz 2016) analysiert. Es wurden vier Oberkategorien gebildet: Raum der Repräsentation, Repräsentationen des Raums, Rolle der Leitung und sozialräumliche Vernetzung. Auf Basis der Netzwerkkarten wurden fallspezifische Kooperationsprofile entlang der sieben Bildungsbereiche und der Querschnittsbereiche der bundeslandspezifischen Bildungsempfehlungen sowie der Typen der Kooperationspartner erstellt. Um die Syntheseleistungen der Leitungskräfte zu erfassen, wurden diese Angaben mit sozialstrukturellen und -kulturellen 
Bedingungen in den Einrichtungen und Sozialräumen (Repräsentationen des Raums) und den Kategorien Raum der Repräsentation, sozialräumliche Vernetzung und Rolle der Leitung verschränkt. Der Raum der Praktiken wurde nicht berücksichtigt, da die Analysen anhand der Interviewdaten erfolgt sind und Beobachtungen o. ä. nicht einbezogen wurden.

\section{Sozialräumliche Vernetzung und die Rolle von Leitungen}

\subsection{Repräsentationen des Raums - sozialstrukturelle und sozialkulturelle Merkmale der Kitas und der Stadtteile}

Die Kitas, die an der vorliegenden Studie teilnehmen, liegen in sechs unterschiedlichen Stadtteilen über vier von sieben Bezirken Hamburgs verteilt. Fünf davon befinden sich in einem Stadtteil mit einem mittleren Statusindex ${ }^{1}$, eine in einem mit einem sehr niedrigen. Die Einrichtungen unterscheiden sich zudem hinsichtlich ihrer Größe (Anzahl Kinder von 34 bis 140; Anzahl Fachkräfte von acht bis 29). Der geringste Anteil von Kindern mit Migrationshintergrund liegt bei 35\%, der höchste bei $98 \%$. Zusätzlich zum Deutschen sprechen die Kinder zwischen sechs und 13 Sprachen. Sprachbildung stellt in allen Kitas einen Schwerpunkt der Arbeit dar, den die Leitungen setzten. Hiermit sind besondere Investitionen der Leitungskräfte in die professionelle Weiterentwicklung der Teams verbunden. Zudem sind in allen Einrichtungen mehrsprachige Fachkräfte tätig. Fünf von sechs Kitas nehmen jeweils an einem städtischen und einem nationalen Programm zur sprachlichen Bildung teil. In beiden Programmen erhalten Kitas mit einem hohen Anteil mehrsprachiger Kin-

Tab. 1 Kooperationen entlang der Bildungs- und Querschnittsbereiche

\begin{tabular}{lll}
\hline & Beispiel & Anzahl Kitas \\
\hline Bildungsbereiche & & \\
Sprachen & Stadtteilbibliothek & 5 \\
Musik & Singpaten & 2 \\
Körper/Bewegung & Schwimmbad & 2 \\
Soziale und kulturelle Umwelt & Kulturzentrum/Bürgerhaus & 5 \\
Natur/Technik & Technikmuseum & 1 \\
Querschnittsbereiche & & \\
Übergang & Grundschule & 4 \\
Bildungs- und Erziehungspartnerschaften & Elternschule & 6 \\
Frühförderung & Ergotherapeutinnen und -therapeuten & 4 \\
Weitere & & \\
& Andere Kitas & 4 \\
& (Regionale) Netzwerke & 2 \\
\hline
\end{tabular}

\footnotetext{
1 Mit dem städtischen Sozialmonitoring werden soziale Merkmale wie der Anteil der Arbeitslosen, der Empfängerinnen und Empfänger von SGB II-Leistungen sowie der Kinder und Jugendlichen mit Migrationshintergrund in den statistischen Gebieten erfasst. Mit dem Statusindex werden die Gebiete in die Kategorien hoch, mittel, niedrig und sehr niedrig eingeteilt.
} 
der und sozial schwachen Familien eine Förderung in Form personeller Ressourcen für sprachliche Bildung.

\subsection{Sozialräumliche Vernetzung}

Die Einrichtungen weisen zwischen fünf und 16 Kooperationspartner in einem breit gefächerten Spektrum auf. Fallübergreifend werden fünf der sieben Bildungsbereiche durch Kooperationen mit externen Partnern vertieft: die Bereiche Sprachen, soziale und kulturelle Umwelt, Natur/Technik sowie Musik und Bewegung. Zudem werden Querschnittsbereiche der Bildungsempfehlungen (Übergang Schule, Bildungs- und Erziehungspartnerschaft und Frühförderung) mit Hilfe von Kooperationspartnern gestaltet. Darüber hinaus kooperieren alle Kitas mit weiteren Partnern (z.B. anderen Kitas). Am häufigsten wird mit Stadtteilbibliotheken, Grundschulen, weiteren Kitas, Therapeutinnen und Therapeuten, Stadtteilzentren und familienunterstützenden bzw. -ergänzenden Einrichtungen (z. B. Elternschule) zusammengearbeitet. Tab. 1 fasst diese Ergebnisse fallübergreifend zusammen.

Die Ergebnisse zeigen, dass die untersuchten Kitas auf vielfältige Weise sozialräumlich vernetzt sind. Ersichtlich wird jedoch auch, dass fallübergreifend weder eine spezifische Fokussierung, die sich in der Anzahl der Kooperationspartner ausdrückt, auf Partner für die migrationsgesellschaftlich relevante Sprachbildung noch Kooperationen mit migrationsspezifischen Einrichtungen bestehen.

Die weitere Analyse förderte zudem zutage, dass es maßgeblich von den Leitungskräften abhängt, welche Kooperationen eingegangen werden. Die Leitungen verstehen sich fallübergreifend als diejenigen, die die Verantwortung für den Aufbau und die Gestaltung der sozialräumlichen Vernetzung tragen. Für ebendiese spielen die internen (sozialstrukturellen und -kulturellen) Bedingungen und die durch die Leitungen wahrgenommenen (Kooperations-)Bedarfe eine Rolle. Im maximalen Kontrast (Kriterium: Anteil Kinder mit Migrationshintergrund) zweier Einrichtungen (Kita Ameise und Kita Fuchs) werden diese Zusammenhänge folgend dargestellt.

\subsection{Raum der Repräsentation und Syntheseleistungen - die Rolle der Leitungen für sozialräumliche Vernetzung}

In Kita Fuchs sind der Anteil von Kindern mit Migrationshintergrund (35\%) und die sprachliche Vielfalt (sechs Sprachen zusätzlich zum Deutschen) im Stichprobenvergleich am geringsten. Die Einrichtung liegt in einem Stadtteil mit mittlerem Statusindex. Die Leitung (LF) bezieht sich in ihrer Beschreibung des Stadtteils auf einen tradierten regionalen und gesellschaftlichen Diskurs, der schon in ihrer Kindheit geführt wurde und der ihre Wahrnehmung bis heute prägt. Sie grenzt den Stadtteil, in dem die Kita liegt, vom Nachbarstadtteil ab, wobei

[..] der andere Stadtteil ist das Gute und dieser ist eher das nicht so Gute. Es sind eher sozialschwache Familien in diesem ansässig gewesen, hohe Arbeitslosigkeit. Sehr viel multi-kulti Geschäfte in der Hauptstraße, das ist ja auch bis heute so geblieben, sämtliche Nationen sind vertreten, dadurch häufig auch Streitereien unter den Nationen. Das hat den Stadtteil immer eher zu einem 
schmuddeligen Stadtteil gemacht, der nicht so sehr in der Gesellschaft angesehen wurde. (Kita Fuchs, 52-58)

In der Wahrnehmung der Leitung erscheint der Stadtteil als problembelastet. Aus migrationsgesellschaftlicher Perspektive ist interessant und gleichsam problematisch, dass die Leitung eine Kausalität (,dadurch“) zwischen unterschiedlichen Herkünften, die im Stadtteil aufeinandertreffen, und Streitigkeiten herstellt.

Allerdings befindet sich der Stadtteil, in dem die Kita liegt, aus Leitungssicht durch (städte-)bauliche Maßnahmen im „Riesenaufschwung“ (63), ,,es passiert [...] ganz viel, dass dieses Schmuddel-Flair wegkommt“ (61-62), und das ,tut diesem Viertel aber GUT“ (39-40). In der Wahrnehmung (Raum der Repräsentation) der Leitung wird so die Reproduktion des hegemonialen Diskurses über den Stadtteil nicht nur ersichtlich, sondern er wird von ihr untermauert.

Von den Veränderungen im Stadtteil profitiert die Kita aus Leitungssicht in der Hinsicht, dass sie hierdurch ein „wunderbar gemischte[s] Klientel“ (33) von „Hartz IV-Empfängern“ (34) bis „Ärzten“ (35) in einem ,ausgewogenen Verhältnis“ (36) hat. Im Hinblick auf den Migrationsanteil bildet sich die Sozialstruktur des Stadtteils jedoch nicht in der Kita ab. So liegt er im Stadtteil bei $61,11 \%$ und in der Kita bei $35 \%$, was die Leitung auf die religiöse bzw. weltanschauliche Ausrichtung der Kita zurückführt, die eine Zugangshürde für Eltern bedeuten könne.

Die Einrichtung erscheint sozial eher gering belastet, was sich beispielsweise darin zeigt, dass sie im Gegensatz zu den anderen fünf Kitas weder an dem städtischen noch an dem nationalen Programm zur sprachlichen Bildung teilnimmt.

Sprachliche Bildung stellt für die Leitung nichtsdestotrotz ein sehr wichtiges Thema dar, dessen Etablierung aus ihrer Sicht jedoch noch am Anfang steht. Gleichwohl werden aufseiten der Kinder und Familien kaum Bedarfe in diesem Bildungsbereich gesehen:

LF: Alles sehr gemischt, wobei die Kinder bis auf zwei Kinder tatsächlich alle in Deutschland geboren sind und teilweise die Eltern auch schon. Also wenig Sprachbarrieren hier in der Kita [...]. (Kita Fuchs, 72-74)

Im Verhältnis zur Gesamtanzahl der Kinder, die die Einrichtung besuchen, und im Vergleich mit den anderen Kitas der Stichprobe besitzen relativ viele Kinder hier einen spezifischen Förderbedarf, weshalb frühe therapeutische Förderung (Ergo-, Logo- und Physiotherapie) aus Leitungssicht ein wichtiges Thema ist. Die wahrgenommene Relevanz dieser Förderung spiegelt sich auch in der sozialräumlichen Vernetzung wider: So bestehen hier mit drei Partnern die meisten Kooperationen der Einrichtung, während sie mit je einer Einrichtung im Querschnittsbereich Bildungs- und Erziehungspartnerschaft und dem Bildungsbereich soziale und kulturelle Umwelt kooperiert. Im Bildungsbereich Sprachen besteht eine Kooperation mit der Stadtteilbibliothek. Deutlich wird demnach der Zusammenhang zwischen den Bedingungen in der Kita, den wahrgenommenen Bedürfnissen der Kinder und der sozialräumlichen Vernetzung.

Im Hinblick auf die sozialräumliche Vernetzung stellt die Leitung heraus, dass sie diejenige ist, die Kooperationen mit Externen aufnimmt und pflegt. 
Kita Ameise weist den höchsten Anteil von Kindern mit Migrationshintergrund (98\%) in der Stichprobe auf. In ihr ist die sprachliche Heterogenität mit elf Sprachen und verschiedenen Dialekten, die zusätzlich zum Deutschen gesprochen werden, mit am größten. Sie erscheint intern am sozial belastetsten und sie liegt gleichzeitig in dem Stadtteil mit dem niedrigsten Statusindex. Die Leitung (LA) schildert den Stadtteil wie folgt:

LA: Hm. Ja. [...]. Wie gesagt ist auch oft in den Medien schon gewesen nicht so positiv. [...] Wir haben natürlich auch schon Probleme gehabt mit Drogen auf dem Spielplatz und solche Sachen, ne? Spritzen gefunden. Aber das ist mal mehr und mal wieder weniger, ne, oder auch hier, was denn hier die Straße oder die Straße geht dann mal durch die Zeitung. Ist nun mal so. Wo viele Menschen aufeinandertreffen und vielleicht auch viele Kulturen, da passiert vielleicht auch viel, ne? Und diese Drogenproblematik, die die Stadt hat, die ist sowieso/ die verschiebt sich immer nur/ wenn sie dann irgendwo aktiv werden, verschiebt die sich in einen anderen Bereich. Aber im Großen und Ganzen ist es friedlich hier, ne? Und eben sehr/ im Sommer. Wenn man hier im Sommer mal durchgeht und es riecht aus allen Fenstern, dann denkt man so: Ach. Ich muss gar nicht in Urlaub fahren. Das ist hier wie im Urlaub, ne? So riecht das hier, ne? Weil die Familien alle was anderes kochen, ne, und dann sind die Fenster auf und die Düfte, das ist schon toll. (Kita Ameise, 55-71)

Die Leitung verweist hier unter anderem auf Probleme, die ihre Ursache auch in städtischen Interventionen haben, und aus ihrer Sicht nicht ein ,Problem des Raums sind'. Sie reproduziert die medial negative Rezeption des Stadtteils und damit den hegemonialen Diskurs nicht, sondern setzt dieser Sicht eine positive entgegen, indem sie feststellt, „dass es im Großen und Ganzen friedlich“ sei und sie „nicht in Urlaub fahren“ müsse, weil es in dem Stadtteil „wie im Urlaub“ rieche.

Wie auch in Kita Fuchs spiegelt sich die Sozialstruktur des Stadtteils in der Kita aus Leitungssicht. So sei das „Publikum“ (182) der Kita ein „gemischtes“ (182), wenngleich „die, die viel Geld haben“ (183) nicht vertreten sind. Ebenso wie im Hinblick auf den Stadtteil benennt sie damit verbundene Herausforderungen, aber auch hier findet sich eine positive Deutung und Offenheit:

LA: Hm. Also einmal, dass es eine Herausforderung ist. Auf jeden Fall. Und dass es auch immer wieder spannend ist. (Kita Ameise, 178-181)

Im Hinblick auf die soziale Lage in der Kita stellt die Leitung dar, dass viele Kinder in ihrer Einrichtung aufgrund der prekären Bedingungen, unter denen sie häufig aufwachsen, im familiären Kontext viele (Bildungs-)Erfahrungen nicht machen können. Zudem bräuchten Familien oft Unterstützung in erzieherischen und weiteren Fragen. So ist es der Leitung besonders wichtig, dass Kinder und Familien auch im Sozialraum die benötigte Unterstützung bekommen:

LA: Dass sie [eine Familie] weiß, wo sie was an Hilfe kriegen kann, wenn sie Hilfe braucht, das finde ich wichtig. (Kita Ameise, 1172-1173)

Deutlich wird, dass es der Leitung um die Erweiterung der Bildungs- und Handlungsmöglichkeiten von Kindern und Eltern geht. Wie in Kita Fuchs stellt sprachli- 
che Bildung für diese Leitung ebenfalls ein wichtiges Thema dar. Im Gegensatz zu Kita Fuchs sieht sie jedoch einen hohen Bedarf in diesem Bildungsbereich, den sie mit dem Anteil mehrsprachiger Kinder begründet, und erachtet dieses Handlungsfeld als gut etabliert in der Kita.

Die Leitung sieht sich - wie auch die Leitung der Kita Fuchs - in der Verantwortung, Kooperationen im Stadtteil einzugehen und zu pflegen:

LA: Ja. Die Kontakte zu knüpfen und aufrechtzuerhalten, ne? Immer wieder am Leben zu halten auch, ne? Und auch zu sehen, dass diese Leute jetzt hier/ sie (sehen?) Paten und die, die ganz freiwillig herkommen, die hier gar nicht (lachend) herkommen müssten, dass die gerne kommen, ne? Das ist schon wichtig ja, [...] dass man auch denen zeigt, dass sie hier sehr willkommen sind und dass es toll ist, was sie hier machen, ne? (Kita Ameise, 1100-1110)

Diese Kita hat die meisten Kooperationspartner in der Stichprobe. Sie kooperiert insbesondere mit Einrichtungen in den Bildungsbereichen Sprachen (drei) und soziale und kulturelle Umwelt (vier) sowie im Bereich Bildungs- und Erziehungspartnerschaft (drei). Auch hier zeigt sich somit ein Zusammenhang mit den Bedingungen in der Kita, den durch die Leitung wahrgenommenen Bedarfen und der sozialräumlichen Vernetzung.

Zusammengefasst veranschaulichen diese beiden Fälle exemplarisch, dass Leitungskräfte aus ihrer Sicht die Verantwortung für die Gestaltung der sozialräumlichen Kooperation tragen. Im Hinblick auf die Syntheseleistungen, also die Verknüpfung von Kita und Instanzen im Stadtteil zu einem Bildungsraum, wurde ersichtlich, dass die Bedingungen in der Kita und ihrem Umfeld sowie die Wahrnehmung der Bedarfe der Kinder (und Familien) eine Basis für das Eingehen von Kooperationen darstellen. Zudem konnte durch die Beleuchtung der Wahrnehmungen der Stadtteile (Raum der Repräsentation) ein unterschiedlicher Umgang mit hegemonialen Diskursen gezeigt werden: In einem Fall eine Reproduktion, im anderen ein Aufbrechen.

\section{Diskussion und Ausblick}

In diesem Beitrag wurde beleuchtet, welche Bedeutung der sozialstrukturellen und sozialkulturellen Zusammensetzung des Stadtteils und der Kita für die sozialräumliche Vernetzung im migrationsgesellschaftlichen Kontext hat und welche Rolle den Leitungskräften hierfür zukommt. Dabei standen die Wahrnehmungen der Leitungskräfte im Mittelpunkt. Aufbauend auf bisherigen theoretischen und empirischen Erkenntnissen wurde angenommen, dass die beteiligten Kitas mit Partnern kooperieren, die im migrationsgesellschaftlichen Kontext relevant sind (z. B. hinsichtlich der sprachlichen Bildung) und dass die Leitungen eine aktive Rolle bei der Vernetzung übernehmen.

Fallübergreifend nehmen sich die Leitungen als diejenigen wahr, die für sozialräumliche Vernetzung Verantwortung tragen. Im Hinblick auf die Syntheseleistungen wird ein Zusammenhang zwischen den Wahrnehmungen der Leitungskräfte, den sozialstrukturellen und -kulturellen Bedingungen in der Kita und der sozialräumlichen Vernetzung ersichtlich: In den Bereichen, in denen die Leitungen Bedarfe der Kin- 
der (und Familien) wahrnehmen, gehen sie auch Kooperationen ein. Sie erscheinen damit als wichtige Akteure, wenn es darum geht, Vernetzung und Kooperation von Kitas zu gestalten, womit bisherige Annahmen und Erkenntnisse zur Bedeutung der Leitungskräfte für dieses Aufgabenfeld von Kitas untermauert werden. Gleichzeitig förderte die Untersuchung zutage, dass fallübergreifend weder eine spezifische Fokussierung auf Partner für die Sprachbildung (als wichtiges migrationsgesellschaftliches Feld frühkindlicher Bildung) noch Kooperationen mit migrationsspezifischen Einrichtungen besteht. Und das, obwohl sowohl die Stadtteile als auch die Kitas als sozialstrukturell und -kulturell heterogen wahrgenommen werden und in den meisten Einrichtungen etwa sprachliche Bildung aufgrund der internen sozialstrukturellen und -kulturellen Bedingungen als wichtiges Handlungsfeld angesehen wird. Warum dies so ist, kann auf Basis der vorliegenden Daten nicht abschließend beantwortet werden. Aber sie lassen Vermutungen zu:

Im Feld der sprachlichen Bildung wird in allen Kitas insbesondere in die Weiterqualifizierung der Fachkräfte selbst investiert. Zudem arbeiten in den Einrichtungen mehrsprachige Fachkräfte. Ihnen wird von den Leitungen teilweise eine sprachlichkulturelle Brückenfunktion zugeschrieben. $\mathrm{Zu}$ vermuten ist, dass aufgrund dieser internen Bedingungen kein erhöhter Bedarf für externe Kooperationen im Bildungsbereich Sprachen gesehen wird. Dieser Vermutung folgend hängen die Syntheseleistungen der Leitungen bei der Konstituierung eines Bildungsraums auch von den internen Ressourcen, hier etwa den Qualifikationen der Fachkräfte, ab.

Zudem liefern die Daten Hinweise darauf, dass die professionell-persönlichen $\mathrm{Zu}$ gänge der Leitungen zum Umgang mit sprachlich-kultureller Diversität bedeutsam sind. Denn einerseits zeigt unsere Analyse, dass die jeweilige Sozialisation der Leitungen und auf dieser Basis angenommene Unterschiede $\mathrm{zu}$ den Lebenswelten von Familien mit Migrationshintergrund das Eingehen von Kooperationsbeziehungen beeinflussen. Andererseits nehmen die Befragten hinsichtlich der Kooperationen auf die je individuellen Bedarfe der Familien bzw. Kinder Bezug, (explizite) Differenzziehungen entlang von Nationen oder Ethnien werden nicht vorgenommen. Diese Leitungsperspektiven könnten dazu beitragen, dass beispielsweise Migrantenselbstorganisationen, die häufig national-ethnisch organisiert sind, nicht als potentielle Kooperationspartner ins Blickfeld rücken.

Inwiefern fehlende Kooperationen mit Partnern für die sprachliche Bildung und mit migrationsspezifischen Einrichtungen bedeutsam für die Gestaltung von Bildungsprozessen in der Kita sind, kann auf Basis der vorliegenden Daten nicht beantwortet werden. Es kann aber konstatiert werden, dass dies ein Indikator dafür ist, dass in Kitas die Diversität von Kindern und Familien diesbezüglich noch nicht ausreichend Berücksichtigung findet.

Im Hinblick auf die Veränderung bestehender gesellschaftlicher Macht- und Ungleichheitsverhältnisse kommt Leitungen - wie bereits ausgeführt - eine zentrale Rolle zu. Einen Beitrag zur Veränderung der gesellschaftlichen Verhältnisse können Leitungen jedoch nur leisten, wenn sie die lokalen wie die gesellschaftlichen Bedingungen im Blick haben, hegemoniale Diskurse hinterfragen, wie es etwa in Kita Ameise ersichtlich wurde, und Syntheseleistungen vollziehen, die bestehenden Ungleichheitsverhältnissen etwas entgegensetzen. So können Bildungsmöglichkeiten eröffnet und erweitert werden, womit ein Beitrag zur Verbesserung von Bildungs- 
chancen ,traditionell benachteiligter Personen' (Hard et al. 2013, S. 328) in der superdiversen Migrationsgesellschaft geleistet werden kann.

Die Syntheseleistungen von Leitungskräften, so lässt sich aus den Untersuchungsergebnissen ableiten, sind demnach bedeutsam für die sozialräumliche Vernetzung von Kitas. Dabei spielen, so ist zu vermuten, nicht nur sozialstrukturelle und -kulturelle Bedingungen in den Einrichtungen und daraus von den Leitungen abgeleitete Bedarfe von Familien und Kindern eine Rolle, sondern auch interne Ressourcen und professionell-persönliche Zugänge. Es wäre weitere Forschung notwendig, die dieser Vermutung nachgeht.

Limitationen der Studie sind, dass sie auf Leitungskräfte fokussierte und dabei unberücksichtigt blieb, inwiefern die Fachkräfte ebenfalls Kooperationsbeziehungen pflegen und damit auch (zusätzliche) Bildungsräume konstituieren. Zudem lagen keine Angaben dazu vor, welche bildungsrelevanten Institutionen im Umfeld der Kita ansässig sind. Dies wäre in künftiger Forschung jedoch zu berücksichtigen, um zu erfassen, welche Möglichkeiten für Kitas bestehen, mit z. B. migrationsspezifischen Instanzen zu kooperieren.

Funding Open Access funding provided by Projekt DEAL.

Open Access Dieser Artikel wird unter der Creative Commons Namensnennung 4.0 International Lizenz veröffentlicht, welche die Nutzung, Vervielfältigung, Bearbeitung, Verbreitung und Wiedergabe in jeglichem Medium und Format erlaubt, sofern Sie den/die ursprünglichen Autor(en) und die Quelle ordnungsgemäß nennen, einen Link zur Creative Commons Lizenz beifügen und angeben, ob Änderungen vorgenommen wurden.

Die in diesem Artikel enthaltenen Bilder und sonstiges Drittmaterial unterliegen ebenfalls der genannten Creative Commons Lizenz, sofern sich aus der Abbildungslegende nichts anderes ergibt. Sofern das betreffende Material nicht unter der genannten Creative Commons Lizenz steht und die betreffende Handlung nicht nach gesetzlichen Vorschriften erlaubt ist, ist für die oben aufgeführten Weiterverwendungen des Materials die Einwilligung des jeweiligen Rechteinhabers einzuholen.

Weitere Details zur Lizenz entnehmen Sie bitte der Lizenzinformation auf http://creativecommons.org/ licenses/by/4.0/deed.de.

\section{Literatur}

Ballaschk, I., \& Anders, Y. (2015). Führung als Thema deutscher Kindertageseinrichtungen. Welchen Beitrag können organisationspsychologische Theorien zur Konzeptentwicklung leisten? Zeitschrift für Pädagogik, 61(6), 876-896.

Bundesministerium für Familie, Senioren, Frauen und Jugend (2020). Kinder- und Jugendhilfe. Achtes Buch Sozialgesetzbuch. Berlin: Bundesministerium für Familie, Senioren, Frauen und Jugend.

Dirks, S., \& Kessl, F. (2012). Räumlichkeit in Erziehungs- und Bildungsverhältnissen. In U. Bauer \& U.H. Bittlingmayer (Hrsg.), Handbuch Bildungs- und Erziehungssoziologie (S. 507-525). Dordrecht: VS.

Dresing, T., \& Pehl, T. (2015). Praxisbuch Interview, Transkription und Analyse. Anleitungen und Regelsysteme für qualitativ Forschende. Marburg: Dr. Dresing und Pehl. www.audiotranskription.de/ praxisbuch

Fröhlich-Gildhoff, K., Nentwig-Gesemann, I., \& Pietsch, S. (2011). Kompetenzorientierung in der Qualifizierung frühpädagogischer Fachkräfte. Eine Expertise der Weiterbildungsinitiative Frühpädagogische Fachkräfte (WiFF). Ausbildung, Bd. 19. München: Dt. Jugendinst..

Hard, L., Press, F., \& Gibson, M. (2013). 'Doing' social justice in early childhood: the potential of leadership. Contemporary Issues in Early Childhood, 14(4), 324-334. 
Hollstein, B., \& Pfeffer, J. (2010). Netzwerkkarten als Instrument zur Erhebung egozentrierter Netzwerke. http://www.pfeffer.at/egonet/Hollstein\%20Pfeffer.pdf. Zugegriffen: 26. Febr. 2020.

Hujala, E. (2013). Contextually defined leadership. In E. Hujala, M. Waniganayake \& J. Rodd (Hrsg.), Researching leadership in early childhood education (S. 47-60). Tampere: University Press.

Jares, L. (2019). Familie und Familienzentren als sozialraumbezogenes Handlungsfeld. In F. Kessl \& C. Reutlinger (Hrsg.), Handbuch Sozialraum. Grundlagen für den Bildungs- und Sozialbereich (2. Aufl. S. 471-486). Wiesbaden: VS.

Jung, E., \& Gels, A. (2019). Vernetzung von KiTas im Sozialraum und darüber hinaus. Nifbe-Beiträge zur Professionalisierung, Bd. 10. Osnabrück: Niedersächsisches Institut für frühkindliche Bildung und Entwicklung.

Kessl, F., \& Reutlinger, F. (2010). Reflexive räumliche Haltung. In F. Kessl \& C. Reutlinger (Hrsg.), Sozialraum. Eine Einführung (2. Aufl. S. 125-133). Wiesbaden: VS.

Kuckartz, U. (2016). Qualitative Inhaltsanalyse. Methoden, Praxis, Computerunterstützung (2. Aufl.). Weinheim: Beltz Juventa.

Löw, M. (2001). Raumsoziologie. Frankfurt am Main: Suhrkamp.

Rathmer, B. A. (2012). Kita und Grundschule: Kooperation und Übergangsgestaltung. Konzeptionen Empirische Bestandsaufnahme - Perspektiven. Internationale Hochschulschriften, Bd. 575. Münster: Waxmann.

Salem, T. (2018). Kooperation zwischen Kita und Grundschule für eine durchgängige Sprachbildung. Münster u. a.: Waxmann.

Salem, T., Braband, J., \& Lengyel, D. (2020). Parental cooperation in early childhood education in Germany-bridging language barriers in multilingual settings. In C. Kirsch \& J. Duarte (eds.), Multilingual approaches for teaching and learning. From acknowledging to capitalizing on multilingualism in European mainstream education. Abingdon: Routledge, pp. 168-185.

Stamm, M., \& Edelmann, D. (2013). Zur pädagogischen Qualität frühkindlicher Bildungsprogramme: Eine Kritik an ihrer ethnozentrischen Perspektive. In M. Stamm \& D. Edelmann (Hrsg.), Handbuch frühkindliche Bildungsforschung (S. 325-341). Wiesbaden: Springer VS.

Stošić, P. (2012). Lokale Bildungsräume zwischen Struktur und Handlung. Tertium Comparationis, 18(1), $12-24$.

Strehmel, P., \& Ulber, D. (2014). Leitung von Kindertageseinrichtungen. Weiterbildungsinitiative Frühpädagogische Fachkräfte, WiFF Expertisen, Bd. 39. München: DJI.

Vertovec, S. (2007). Super-diversity and its implications. Ethnic and Racial Studies, 30(6), 1024-1054. 